\title{
Human walking dynamics: modeling, identification and control
}

\author{
W. Schiehlen \\ Institute of Engineering and Computational Mechanics, University of Stuttgart, 70550 Stuttgart, Germany
}

\begin{abstract}
Human gait simulation is a complex dynamical problem that requires, in addition to the mechanical model, the observance of muscle activations, neural excitations, and energetic and aesthetic considerations. After an short review on the historical development two- and three-dimensional models using multibody system dynamics are presented. The identification of the muscle actuation during human walking is based on data in literature comparing the resultant torques to each other. The control design uses inverse dynamics approaches and an optimization framework minimizing the metabolical energy consumption and improving the aesthetics. Numerical simulation results are shown for planar as well as spatial models.
\end{abstract}

\section{Introduction}

Human walking dynamics is based on a huge body of research results documented by monographs, textbooks and various journals. It is an important research topic for biomechanics including structural nonlinear dynamics, too. Multibody system dynamics as well as advanced simulation technologies provide many tools supporting human walking research.

This paper is organized as follows. In Chap. 2 early developments are summarized. Chap. 3 is devoted to mechanism models. Chap. 4 presents contributions to parameter identification and optimization while Chap. 5 shows some recent research results on gait simulations as exampels.

\section{Early developments}

A first contribution to human walking is due to the Greek philosopher Aristotle (384-322 BC). His book written in Greek language is entitled "De Motu Animalium". Aristotle made observations of animals and humans, he stated many questions but he did not give scientifically satisfactory answers.

In the Middle Ages a book with the same title "De Motu Animalium" was written with two parts by the Italian mathematician Giovanni Alfonso Borelli (1608 1679) in Latin language. The first part deals with the mechanical motions actuated by the muscles while the second part presents a description of the muscle contraction. Therefore, the book is generally considered as the very first treatise on biomechanics.
The famous book "Mechanik der menschlichen Gehwerkzeuge" was co-authored by two German brothers, the physicist Wilhelm Eduard Weber (1804 1891) and the physician Eduard Friedrich Weber (1806 1871). This book characterizes an interdisciplinary approach to the science of human walking and running. The human body has been divided in carrying and supporting parts where the legs are modelled like pendula swinging at the trunk. Nevertheless, formulae haven't been used.

More details on these early contributions and complete references can be found in [1].

\section{Mechanism models}

A theoretical foundation for mechanics of the living body was presented by the German physicist and mathematician Otto Fischer (1861-1916) in his book [2] For the modelling of human walking Fischer used planar three-link systems and spatial n-link systems. He reduced the complexity of the equations of motion by introducing barycenters and centers of mass. The barycenter approach is explained in detail by Wittenburg [3] while Fischer used mechanical mechanisms to evaluate the human body's overall center of mass. Furthermore, Fischer applied inverse dynamics on a right leg system for the evaluation of the torque generated by the muscles. Thus, Fischer introduced mechanism theory to biomechanics. Some of his important results are summarized as follows:

- The swing motion of the leg is not only a free pendulum oscillation but a motion actuated by muscles. 
- The computed muscle torque does not identify the muscles directly involved in the different phases of the leg motion.

- Fischer suggested only that most probably the muscles Iliopsoas, Rectus Femoris and Tibialis Anterior are initiating the swing phase and their contraction disappear after one third of the swing period.

Complex mechanism models for human walking research are recently designed in multibody system dynamics, see e.g. Silva and Ambrosio [4] and Chenut, Fisette and Samin [5]. For sophisticated models the commercial software AnyBody [6] developed by Rasmussen can be used. Figures 1, 2 and 3 show planar, spatial and commercial models with different complexity.

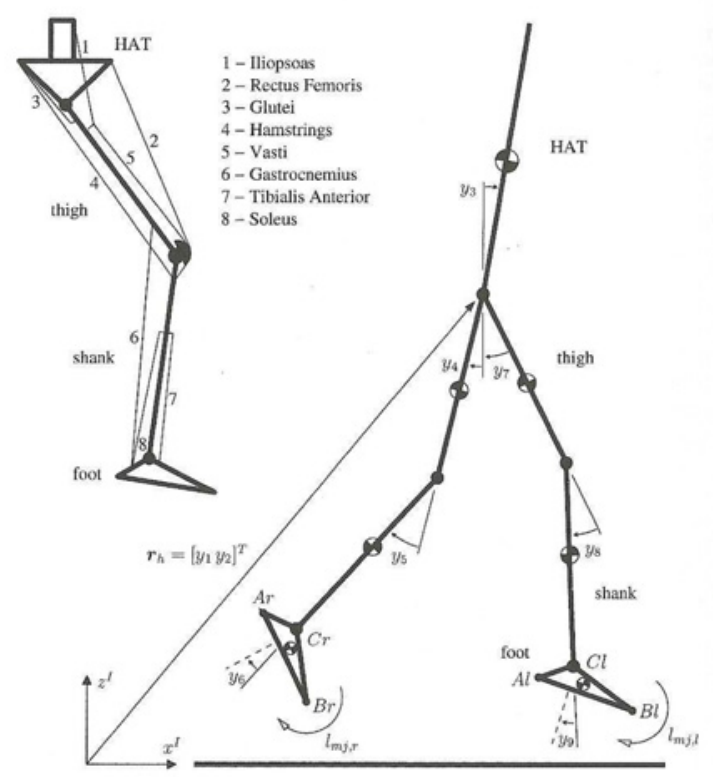

Fig. 1. Planar mechanism model and related muscles [7].

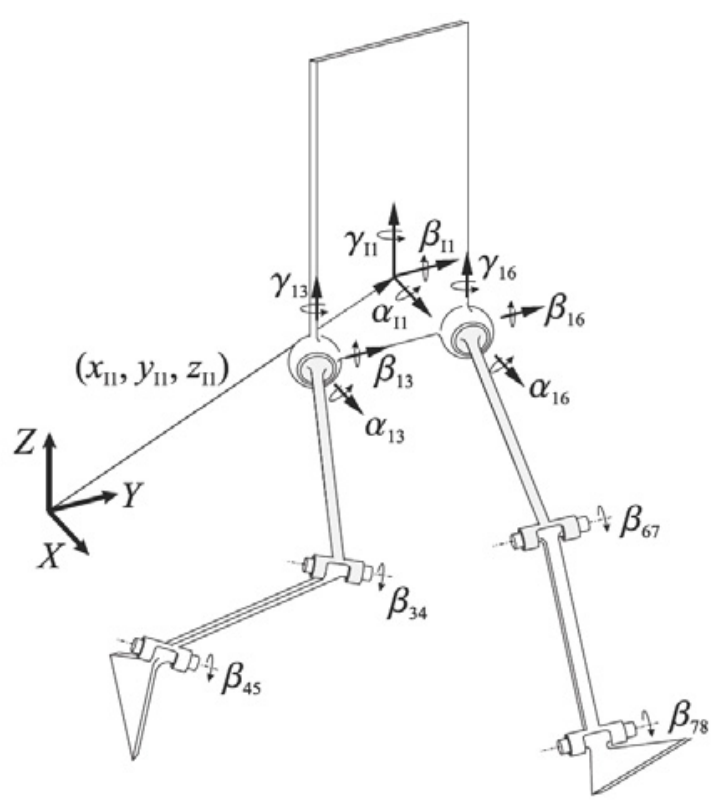

Fig. 2. Spatial mechanism model [8].

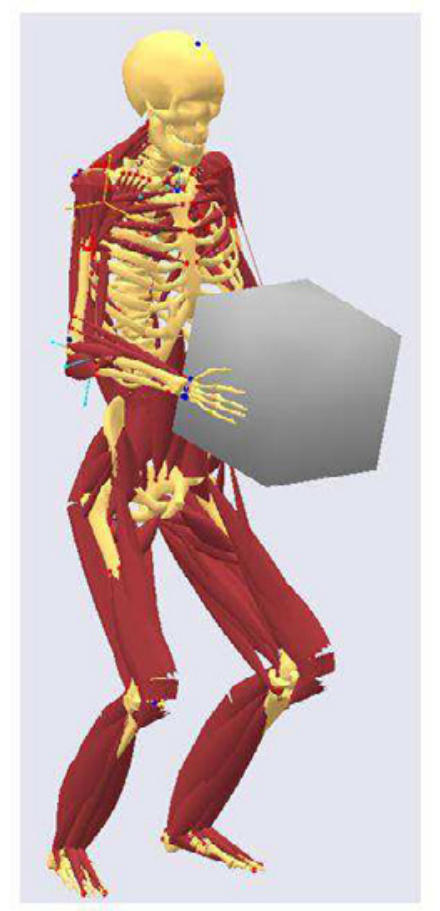

Fig. 3. Anybody mechanism model [6].

The kinematic tree in Fig. 2 is described by the following vector of 16 generalized coordinates

$$
\begin{aligned}
q=\left[\begin{array}{lllllllll}
x_{I 1} & y_{I 1} & z_{I 1} & \alpha_{I 1} & \beta_{I 1} & \gamma_{I 1} & \alpha_{13} & \beta_{13} & \gamma_{13} \\
& \beta_{34} & \beta_{45} & \alpha_{16} & \beta_{16} & \gamma_{16} & \beta_{67} & \beta_{78}
\end{array}\right]^{T}
\end{aligned}
$$

as shown in [8] where the subscript $I$ refers to the inertial frame, subscript 1 refers to body HAT, which is in composed of the pelvis and the trunk, subscripts 3 and 6 refer to right and left thighs, respectively, subscripts 4 and 7 refer to right and left shanks, respectively, and subscripts 5 and 8 refer to right and left feet, respectively. When a subscript is written as $i j$, it means a relative motion of body $j$ with respect to body $i$. It shall be noted here that the notation is based on the most common sequence of rotation 123, while in biomechanics the sequence 213 is usually considered anatomically meaningful, However, while the spatial rotations of the members are the same using the different rotation sequences, for comparison with other authors' results, the 213 sequence has been used.

Once the kinematic tree representing the skeleton is described, the contact of this chain with the ground is added. The contact conditions in the different walking phases are represented by unilateral constraints. However, due to the use of an optimization framework in which it is possible to constrain the normal contact forces to be only positive, the contact with the ground is simply modeled using bilateral constraints associated with the joints attached to the feet. Therefore, the contact forces can be easily added to the model by using Lagrange multipliers. 
$M(q) \ddot{q}+k(q, \dot{q})=q_{r}(q, \dot{q})+B A f^{m}+C_{p h}^{T} \lambda_{p h}$

Here $\lambda p h$ is the vector of Lagrange multipliers at the 8 phase $p h$ of the motion. Furthermore. $\boldsymbol{M}$ is the inertia matrix of the system, $\boldsymbol{k}$ is the vector describing the generalized Coriolis forces, $\boldsymbol{q}_{\boldsymbol{r}}$ is the vector including generalized gravitational forces, passive generalized moments at the joints due to tissues interacting with the joints as well as generalized viscous damping torques at the knees and hips, and $\boldsymbol{B} \boldsymbol{A} \boldsymbol{f}^{\boldsymbol{m}}$ is a vector that includes the generalized forces exerted by the muscles actuating the model.

\section{Parameter identification and optimization}

Since the muscle forces cannot be measured by engineering means, a standard approach is the measurement of the human walking by motion capture systems and to apply inverse dynamics. Then, for the fully parameterized model the forces required for walking are computed. For the validation of the whole procedure inverse and direct dynamics are combined. Then, the muscle forces are the control input for the time integration of the equations of motion. This design principle for nonlinear control problems is well accepted for engineering systems, e.g. in robot control,and for walking machines [9].

However, the muscle forces are generated by activation and contraction dynamics which have to be considered, too. For this purpose, the mechanical model of the walker, Eq. (2), is extended resulting in a larger dynamical system including the eigendynamics of the muscles. The input variable is now the neural excitation which can be found by an extended inverse dynamics (EID) depicted in Figure 4.
For the validation of the whole procedure inverse and direct dynamics are combined as shown in Figs. 5 and 6.

\section{Extended Inverse Dynamics}

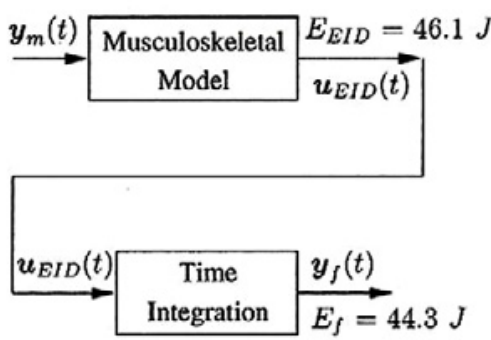

Direct dynamics

Fig. 5. Identification and validation of the neural excitation of the muscle forces [7]

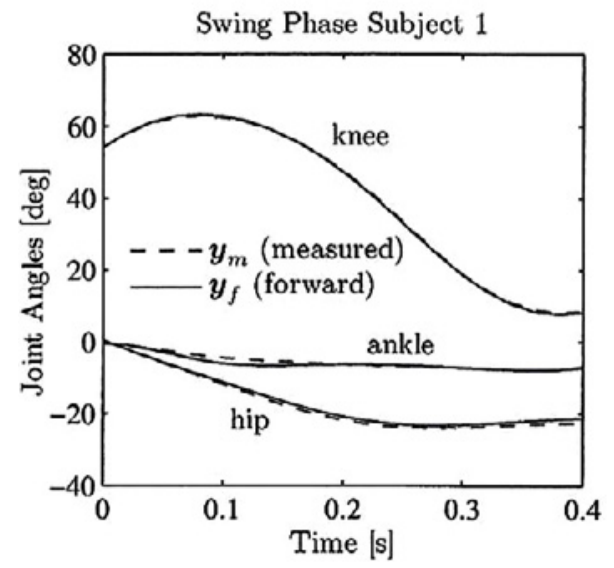

Fig. 6. Comparison of measured kinematics and the computed motion [7].

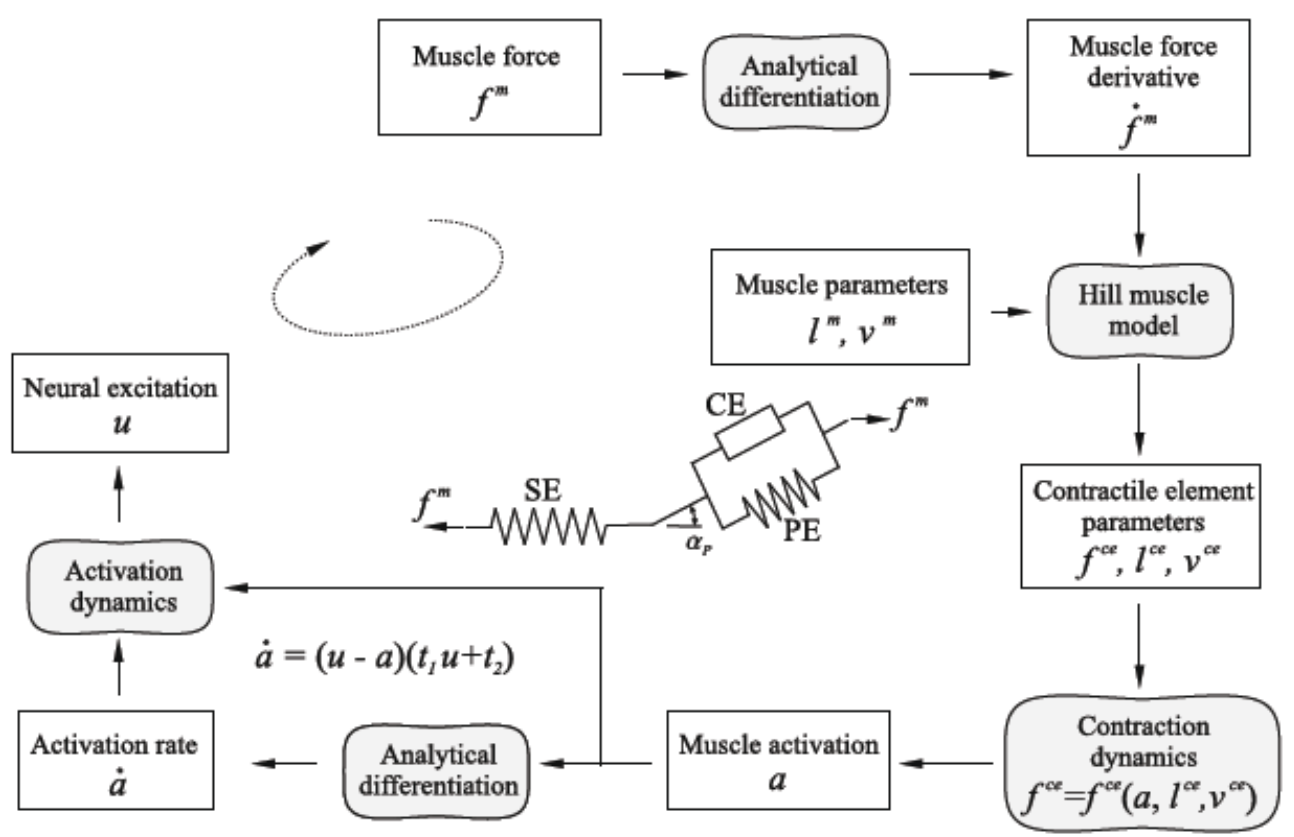

Fig. 4. Extended inverse dynamics [8] 
Inverse dynamics as well as extended inverse dynamics depend on the quality of the model of the dynamical system. In engineering usually the number of degrees of freedom agrees with the number of actuators for unique system controllability. But in biomechanics of walking there are much more muscle actuators resulting in an overactuated system. Therefore, the control design may be improved by an optimization approach if there are suitable criteria at hand. For human walking powerful criteria are given by the expenditure of the metabolical energy during steady-state walking and the aesthetics of walking characterized by the kinematical deviation from normal walking pattern. Both criteria can be weighted and the resulting cost function should be minimized what is however computationally very expensive.

Moreover, human model parameters have some uncertainty since living beings cannot be disassembled like machines. Therefore, the efficiency of the optimization approach is improved by identification of a reduced number of muscles which are mainly actuated by the walking pattern chosen.

\section{Some simulation results}

The optimization problem described in Chapter 4 has been solved by Sequential Quadratic Programming (SQP) from MATLAB Optimization Toolbox for planar models [7] and spatial models [8].

\subsection{Planar model}

First of all a planar walker as shown in Figure 1 is considered. This model has 9 generalized coordinates and 16 actuated muscles. The motion looks aesthetic and the ground reaction forces are also shown, Figure 7 . The quality of the model is very satisfactory as depicted in Figure 8 where measured and simulated motions of the lower limb are presented.

The numerical results obtained for the ground reaction forces are shown in Figure 9 together with the measured data and their standard deviation. The computation time has been more or less 24 hours.

\subsection{Spatial model}

In this section, the three-dimensional non-symmetrical (3DNS) model is studied, see Figure 2. The 3DNS model has 16 generalized coordinates, Eq. (2), and 28 actuated muscles. In order to keep the computation time as small as possible large discretization steps are used for each time dependent variable. Nevertheless, the optimization algorithm used took up to 65 hours to find a solution within the tolerances given. The constraint violation required the scaling of constraints and design variables to ensure good accuracy of the constraint satisfaction.

In this case, the measured motion has been used as a reference motion or normal walking pattern, respectively.

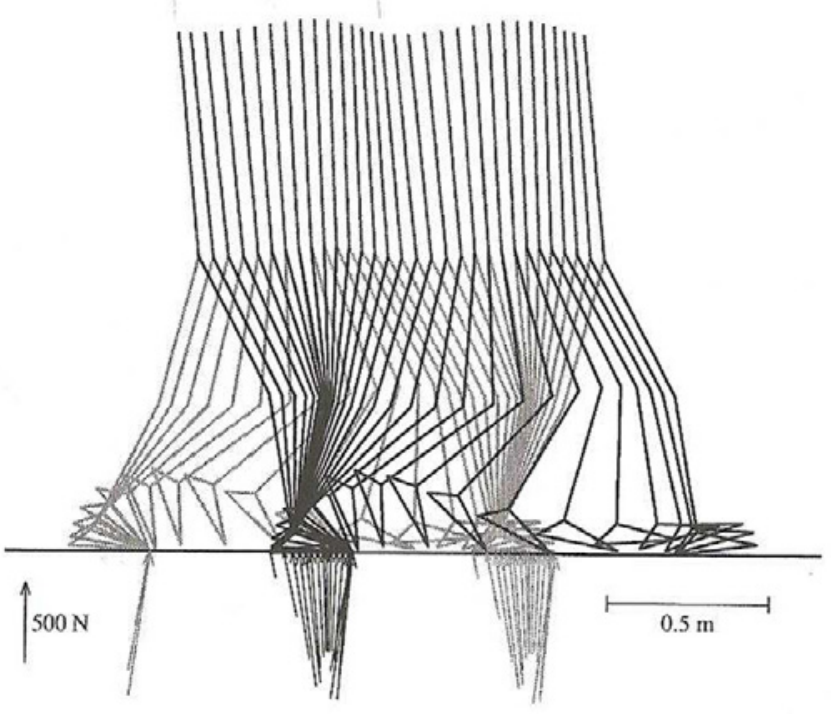

Fig. 7. Stick figure of gait cycle for normal walking [7]

Figure 10 shows the time histories of the three components of the ground reaction forces at both feet. The similarity between the solutions with and without a weight of $2 \mathrm{~kg}$ attached to the right foot is remarkable. The solutions are almost coinciding because of the ability of the three-dimensional non-symmetrical model to accept minor variations of its variables to absorb the effect of a one-sided disorders by the $2 \mathrm{~kg}$ weight. As shown in this section, The 3DNS model provides much richer information of the dynamics of human gait even with one-sided disorders. walking pattern chosen.

\section{Conclusions}

Human walking research has a long tradition in science starting in the Acient times with philosophy and continuing in the Middle Ages by physiology and medicine. Today, human walking research includes nonlinear dynamics of multibody system due to the large rotations of the extremities, biomechanical modelling of muscles as well as the diagnosis of their activation by neural excitations, identification of system parameters and nonlinear control of motions by extended inverse dynamics. Finally, the overactuated dynamical system is treated as an overall optimization problem subject to minimum metabolical energy consumption during steadystate walking and minimal deviation from normal walking for aesthetic reasons. The simulations of forces and motions are computationally very costly, for walking in the sagittal plane it may take up to one day while for spatial walking in three dimension one has to spend several days. The results have been applied to different prosthetic feet, the design of which can be supported by interdisciplinary research reported based on advanced modelling and control techniques. 

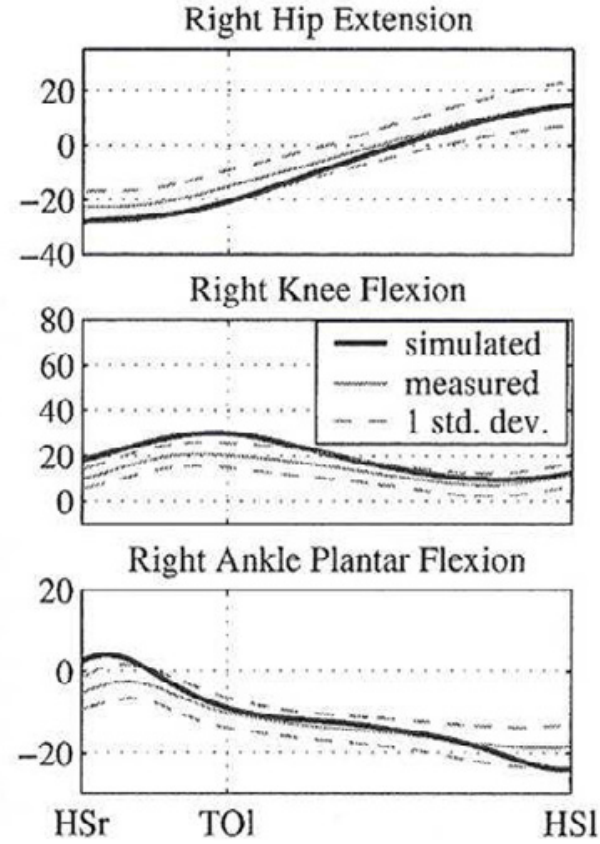
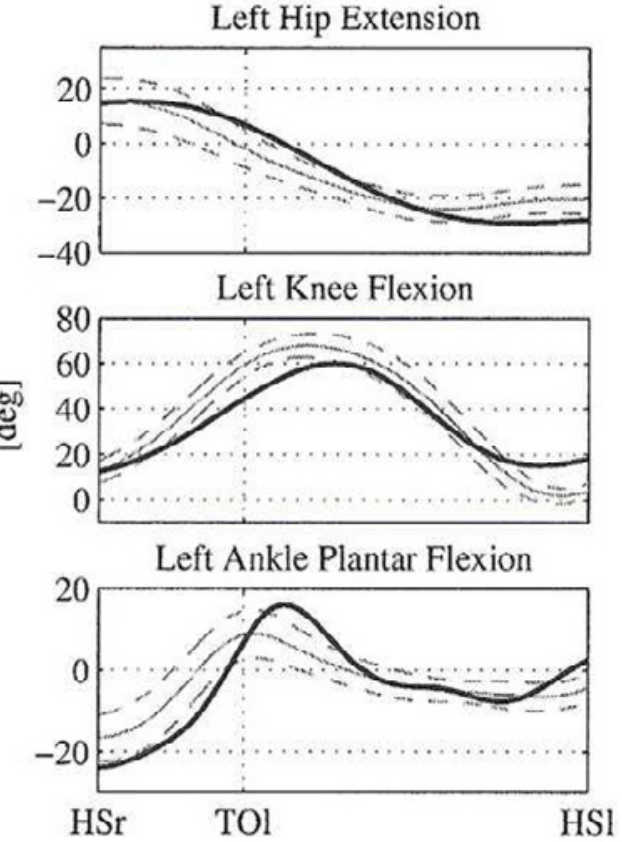

Fig. 8. Measured and simulated motions of lower limb [7]

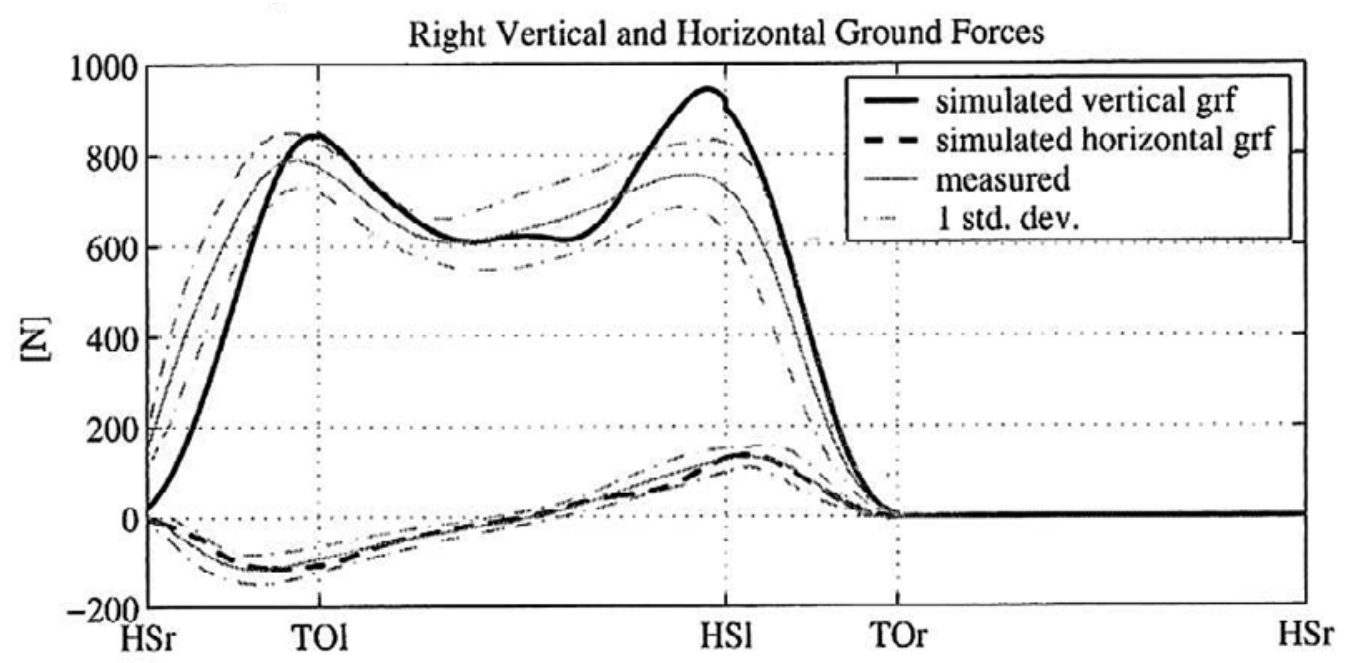

Fig. 9. Measured and simulated ground reaction forces of right foot during planar motion [7] 

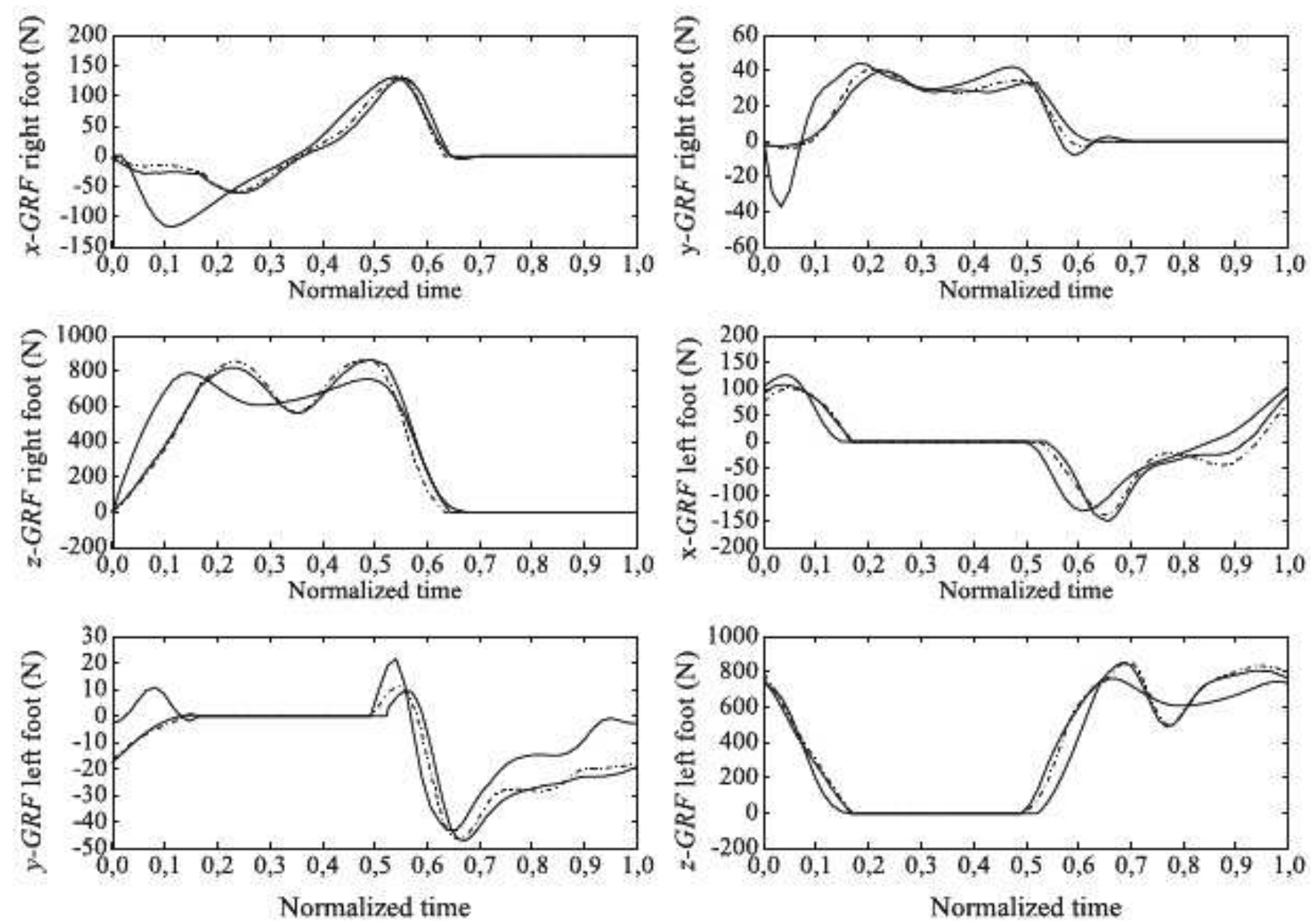

Fig. 10. Ground reaction forces of both feet during spatial motion: measured (solid thin line), simulated (solid thick line) and simulated with gait disorder (dahed thick line) [8]

\section{References}

1. W. Schiehlen, On the historical development of human walking dynamics, in E. Stein (ed.) The history of theoretical, material and computational mechanics 101116 (Springer, Berlin 2014)

2. O. Fischer, Theoretische Grundlagen fuer eine Mechanik der lebenden Körper (Teubner, Leipzig 1906, in German)

3. J. Wittenburg, Dynamics of multibody systems (Springer, Berlin 2008)

4. M.P.T. Silva, J.A.C. Ambrosio, Multibody System Dynamics 8 219-239 (2002)

5. X. Chesnut, P. Fisette, J.C. Samin, Multibody System Dynamics 8 117-140 (2002)

6. AnyBody Technology, AnyBody Modeling System ${ }^{\mathrm{TM}}$ (www.anybodytech.com $>$ Company $>$ Press 2014)

7. M. Ackermann, Dynamics and energetics of walking with prosthesis (Shaker, Aachen 2007)

8. D. Garcia-Vallejo, W. Schiehlen, Arch. Appl. Mech. 82 533-556 (2012

9. S. Gruber, Zur Dynamik und Regelung zweibeiniger Gehmaschinen (VDI Verlag, Düsseldorf 2004, in German) 